Supplement of

\title{
Coupling of organic and inorganic aerosol systems and the effect on gas-particle partitioning in the southeastern US
}

Havala O. T. Pye et al.

Correspondence to: Havala O. T. Pye (pye.havala@epa.gov)

The copyright of individual parts of the supplement might differ from the CC BY 4.0 License. 
Table S1: Functional group assignments of organic compounds and factors used as species in AIOMFAC. AIOMFAC does not include experimentally-constrained interaction parameters for the bisulfate anion with ester, aldehyde, ketone, or aromatic carbon-alcohol functional groups (Zuend and Seinfeld, 2012), although an analogy approach can be employed to estimate these interactions. In addition, organonitrate -ion interaction parameters are not yet available. When needed, these functional groups were assigned to another representative group. Isoprene-OA used in AIOMFAC consisted of measured Isoprene-OA minus explicitly represented isoprene-derived compounds. LO-OOA used in AIOMFAC consisted of measured LO-OOA minus explicitly represented monoterpene-derived compounds. BBOA used in AIOMFAC consisted of measured BBOA minus levoglucosan. For AMS PMF factors, functional group assignments were made by selecting a compound representative of the factor (levoglucosan for BBOA, 2-methyltetrol dimer for Isoprene-OA, $\mathrm{C}_{8} \mathrm{O}_{4} \mathrm{H}_{14}$ for $\mathrm{LO}-\mathrm{OOA}$, and fulvic acid for MO-OOA) and adjusting the functional groups up or down to result in an overall O:C and $\mathrm{H}: \mathrm{C}$ more consistent with the PMF factor. Molecular masses were kept below $500 \mathrm{~g} \mathrm{~mol}^{-1}$. All compound/factor concentrations were set $\geq$ zero and the total mass normalized to reproduce total organic aerosol mass measured by the GT AMS.

\begin{tabular}{|c|c|c|c|c|c|c|c|c|c|c|c|c|c|c|c|c|c|}
\hline \multicolumn{6}{|c|}{ AIOMFAC Functional Groups } & \multicolumn{12}{|c|}{ Number of Functional Groups in Organic Species } \\
\hline \multicolumn{2}{|c|}{ Group Name } & 竘 & $\begin{array}{l}0 \\
0 \\
\vdots \\
\Xi \\
0 \\
0\end{array}$ & $\begin{array}{l}0 \\
0 \\
0 \\
\vdots \\
\vdots \\
0 \\
.10\end{array}$ & $\begin{array}{l}\text { בे } \\
0 \\
\vdots \\
\Xi \\
\pm \\
\end{array}$ & & & 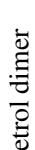 & $\overleftarrow{6}$ & & $\overline{0}$ & & $\stackrel{\overline{\mathrm{O}}}{\mathrm{E}}$ & 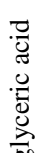 & Е & & 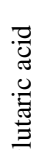 \\
\hline \multirow[t]{4}{*}{$\begin{array}{c}\text { alkyl } \\
\text { (standard) }\end{array}$} & $(\mathrm{CH} 3)$ & 15 & 1 & 0 & 3 & 0 & 0 & 1 & 2 & 2 & 0 & 2 & 1 & 1 & 0 & 3 & 0 \\
\hline & $(\mathrm{CH} 2)$ & 14 & 1 & 0 & 2 & 2 & 1 & 0 & 1 & 1 & 0 & 2 & 0 & 0 & 0 & 2 & 2 \\
\hline & $(\mathrm{CH})$ & 13 & 1 & 0 & 1 & 0 & 1 & 0 & 1 & 1 & 0 & 2 & 0 & 0 & 1 & 2 & 0 \\
\hline & (C) & 12 & 1 & 0 & 0 & 0 & 0 & 1 & 1 & 0 & 0 & 1 & 0 & 0 & 0 & 1 & 0 \\
\hline \multirow[t]{4}{*}{$\begin{array}{c}\text { alkyl in } \\
\text { alcohols }\end{array}$} & $(\mathrm{CH} 3[\mathrm{alc}])$ & 15 & 1 & 0 & 3 & 0 & 0 & 1 & 0 & 0 & 1 & 0 & 0 & 0 & 0 & 0 & 0 \\
\hline & $(\mathrm{CH} 2[\mathrm{alc}])$ & 14 & 1 & 0 & 2 & 0 & 0 & 0 & 0 & 0 & 0 & 0 & 0 & 0 & 0 & 0 & 0 \\
\hline & $(\mathrm{CH}[\mathrm{alc}])$ & 13 & 1 & 0 & 1 & 0 & 0 & 0 & 0 & 0 & 0 & 0 & 0 & 0 & 0 & 0 & 0 \\
\hline & (C[alc]) & 12 & 1 & 0 & 0 & 0 & 0 & 0 & 0 & 0 & 0 & 0 & 0 & 0 & 0 & 0 & 0 \\
\hline \multirow[t]{4}{*}{$\begin{array}{c}\text { alkyl in } \\
\text { tail of } \\
\text { alcohols }\end{array}$} & $\begin{array}{l}(\mathrm{CH} 3[\text { alc- } \\
\text { tail] })\end{array}$ & 15 & 1 & 0 & 3 & 0 & 0 & 0 & 0 & 0 & 0 & 0 & 0 & 0 & 0 & 0 & 0 \\
\hline & $\begin{array}{l}(\mathrm{CH} 2[\text { alc- } \\
\text { tail] })\end{array}$ & 14 & 1 & 0 & 2 & 0 & 0 & 0 & 0 & 0 & 0 & 0 & 0 & 0 & 0 & 0 & 0 \\
\hline & $\begin{array}{l}\text { (CH[alc- } \\
\text { tail] })\end{array}$ & 13 & 1 & 0 & 1 & 0 & 0 & 0 & 0 & 0 & 0 & 0 & 0 & 0 & 0 & 0 & 0 \\
\hline & $\begin{array}{l}\text { (C[alc- } \\
\text { tail]) }\end{array}$ & 12 & 1 & 0 & 0 & 0 & 0 & 0 & 0 & 0 & 0 & 0 & 0 & 0 & 0 & 0 & 0 \\
\hline \multirow{4}{*}{$\begin{array}{c}\text { alkyl } \\
\text { bonded to } \\
\text { OH (OH } \\
\text { separately) }\end{array}$} & $\begin{array}{l}(\mathrm{CH} 3 \\
[\mathrm{OH}])\end{array}$ & 15 & 1 & 0 & 3 & 0 & 0 & 0 & 0 & 0 & 0 & 0 & 0 & 0 & 0 & 0 & 0 \\
\hline & $\begin{array}{l}(\mathrm{CH} 2 \\
[\mathrm{OH}])\end{array}$ & 14 & 1 & 0 & 2 & 1 & 0 & 3 & 1 & 2 & 2 & 0 & 2 & 1 & 0 & 0 & 0 \\
\hline & $(\mathrm{CH}[\mathrm{OH}])$ & 13 & 1 & 0 & 1 & 3 & 2 & 2 & 1 & 1 & 1 & 0 & 0 & 0 & 3 & 1 & 1 \\
\hline & $(\mathrm{C}[\mathrm{OH}])$ & 12 & 1 & 0 & 0 & 4 & 0 & 1 & 0 & 1 & 1 & 0 & 0 & 1 & 0 & 0 & 0 \\
\hline \multirow[t]{3}{*}{ alkenyl } & $(\mathrm{CH} 2=\mathrm{CH})$ & 27 & 2 & 0 & 3 & 0 & 0 & 0 & 0 & 0 & 0 & 0 & 0 & 0 & 0 & 0 & 0 \\
\hline & $(\mathrm{CH}=\mathrm{CH})$ & 26 & 2 & 0 & 2 & 0 & 0 & 0 & 0 & 0 & 0 & 0 & 0 & 0 & 0 & 0 & 0 \\
\hline & $(\mathrm{CH} 2=\mathrm{C})$ & 26 & 2 & 0 & 2 & 0 & 0 & 0 & 0 & 0 & 0 & 0 & 0 & 0 & 0 & 0 & 0 \\
\hline
\end{tabular}




\begin{tabular}{|c|c|c|c|c|c|c|c|c|c|c|c|c|c|c|c|c|c|}
\hline & $(\mathrm{CH}=\mathrm{C})$ & 25 & 2 & 0 & 1 & 0 & 0 & 0 & 0 & 0 & 0 & 0 & 0 & 0 & 0 & 0 & 0 \\
\hline & $(\mathrm{C}=\mathrm{C})$ & 24 & 2 & 0 & 0 & 0 & 0 & 0 & 0 & 0 & 0 & 0 & 0 & 0 & 0 & 0 & 0 \\
\hline \multirow{2}{*}{$\begin{array}{l}\text { aromatic } \\
\text { hydro- } \\
\text { carbon }\end{array}$} & $(\mathrm{ACH})$ & 13 & 1 & 0 & 1 & 0 & 0 & 0 & 0 & 0 & 0 & 0 & 0 & 0 & 0 & 0 & 0 \\
\hline & (AC) & 12 & 1 & 0 & 0 & 0 & 0 & 0 & 0 & 0 & 0 & 0 & 2 & 0 & 0 & 0 & 0 \\
\hline $\begin{array}{c}\text { aromatic } \\
\text { carbon- } \\
\text { alcohol }\end{array}$ & $(\mathrm{ACOH})$ & 29 & 1 & 1 & 1 & 0 & 0 & 0 & 0 & 0 & 0 & 0 & 0 & 0 & 0 & 0 & 0 \\
\hline hydroxyl & $(\mathrm{OH})$ & 17 & 0 & 1 & 1 & 8 & 2 & 6 & 2 & 4 & 4 & 0 & 3 & 2 & 3 & 1 & 1 \\
\hline \multirow[t]{2}{*}{ carboxyl } & $(\mathrm{COOH})$ & 45 & 1 & 2 & 1 & 2 & 0 & 0 & 2 & 0 & 0 & 2 & 0 & 1 & 0 & 1 & 2 \\
\hline & $(\mathrm{HCOOH})$ & 46 & 1 & 2 & 2 & 0 & 0 & 0 & 0 & 0 & 0 & 0 & 0 & 0 & 0 & 0 & 0 \\
\hline \multirow[t]{2}{*}{ ketone } & (CH3CO) & 43 & 2 & 1 & 3 & 0 & 0 & 0 & 0 & 0 & 0 & 0 & 0 & 0 & 0 & 0 & 0 \\
\hline & $(\mathrm{CH} 2 \mathrm{CO})$ & 42 & 2 & 1 & 2 & 0 & 0 & 0 & 0 & 0 & 0 & 0 & 0 & 0 & 0 & 0 & 0 \\
\hline aldehyde & $\begin{array}{l}\mathrm{CHO} \\
\text { [aldehyde] })\end{array}$ & 29 & 1 & 1 & 1 & 0 & 0 & 0 & 0 & 0 & 0 & 0 & 0 & 0 & 0 & 0 & 0 \\
\hline \multirow[t]{2}{*}{ ester } & $\begin{array}{l}(\mathrm{CH} 3 \\
\mathrm{COO})\end{array}$ & 59 & 2 & 2 & 3 & 0 & 0 & 0 & 0 & 0 & 0 & 0 & 0 & 0 & 0 & 0 & 0 \\
\hline & $\begin{array}{l}(\mathrm{CH} 2 \\
\mathrm{COO})\end{array}$ & 58 & 2 & 2 & 2 & 0 & 0 & 0 & 0 & 0 & 0 & 0 & 0 & 0 & 0 & 0 & 0 \\
\hline \multirow[t]{3}{*}{ ether } & $(\mathrm{CH} 3 \mathrm{O})$ & 31 & 1 & 1 & 3 & 0 & 0 & 0 & 0 & 0 & 0 & 0 & 0 & 0 & 0 & 0 & 0 \\
\hline & $(\mathrm{CH} 2 \mathrm{O})$ & 30 & 1 & 1 & 2 & 1 & 1 & 1 & 1 & 0 & 0 & 0 & 0 & 0 & 1 & 0 & 0 \\
\hline & $\begin{array}{l}\mathrm{CHO} \\
\text { [ether] })\end{array}$ & 29 & 1 & 1 & 1 & 1 & 1 & 0 & 0 & 0 & 0 & 0 & 0 & 0 & 1 & 0 & 0 \\
\hline
\end{tabular}

Table S2: Properties of AIOMFAC surrogates.

\begin{tabular}{|c|c|c|c|c|c|c|c|c|c|c|c|c|}
\hline & $\begin{array}{l}\mathbb{1} \\
0 \\
0 \\
0 \\
\vdots \\
\Sigma\end{array}$ & 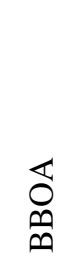 & 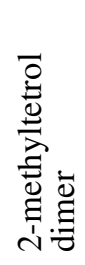 & 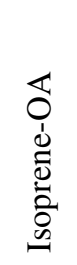 & $\begin{array}{l}\nwarrow \\
0 \\
0 \\
1 \\
0\end{array}$ & 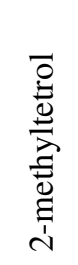 & 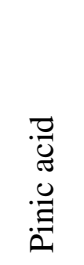 & 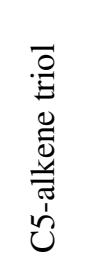 & 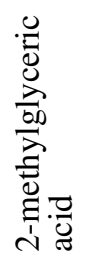 & 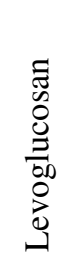 & 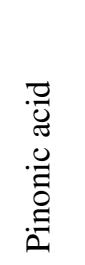 & 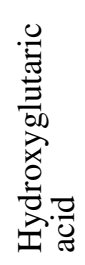 \\
\hline $\begin{array}{c}\text { Molecular } \\
\text { weight } \\
(\mathrm{g} / \mathrm{mol})\end{array}$ & 414 & 146 & 254 & 250 & 178 & 136 & 186 & 118 & 120 & 162 & 186 & 148 \\
\hline $\mathrm{O}: \mathrm{C}$ & 1.00 & 0.67 & 0.70 & 0.70 & 0.50 & 0.80 & 0.44 & 0.60 & 1.00 & 0.83 & 0.30 & 1.00 \\
\hline $\mathrm{H}: \mathrm{C}$ & 1.57 & 1.67 & 2.20 & 1.80 & 2.25 & 2.40 & 1.56 & 2.00 & 2.00 & 1.67 & 1.80 & 1.60 \\
\hline $\mathrm{OM} / \mathrm{OC}$ & 2.46 & 2.03 & 2.12 & 2.08 & 1.85 & 2.27 & 1.72 & 1.97 & 2.50 & 2.25 & 1.55 & 2.47 \\
\hline
\end{tabular}


Table S3: SMILES strings for organic compounds and factors.

\begin{tabular}{|l|l|}
\hline Model Species & SMILES representation \\
\hline MO-OOA & $\mathrm{C} 1(\mathrm{C}(\mathrm{C}(\mathrm{C}(\mathrm{C} 2 \mathrm{C} 1 \mathrm{C}(\mathrm{C} 3(\mathrm{C}(\mathrm{O} 2)(\mathrm{C}(\mathrm{C}(\mathrm{OC} 3)(\mathrm{CO}) \mathrm{O}) \mathrm{O}) \mathrm{O}) \mathrm{O})=\mathrm{O}) \mathrm{O}) \mathrm{O}) \mathrm{O})(\mathrm{C}(=\mathrm{O}) \mathrm{O}) \mathrm{O}$ \\
\hline BBOA & $\mathrm{C} 1 \mathrm{C} 2 \mathrm{C}(\mathrm{CC}(\mathrm{C}(\mathrm{O} 1) \mathrm{O} 2) \mathrm{O}) \mathrm{O}$ \\
\hline Isoprene-OA & $\mathrm{C}(=\mathrm{O})(\mathrm{O}) \mathrm{C}(\mathrm{C}) \mathrm{C}(\mathrm{O}) \mathrm{COC}(\mathrm{C})(\mathrm{CO}) \mathrm{CC}(=\mathrm{O}) \mathrm{O}$ \\
\hline LO-OOA & $\mathrm{CC}(\mathrm{C}) \mathrm{CC}(\mathrm{O})(\mathrm{CO}) \mathrm{C}(\mathrm{O}) \mathrm{CO}$ \\
\hline 2-methyltetrol (monomer) & $\mathrm{C}(\mathrm{O}) \mathrm{C}(\mathrm{O})(\mathrm{C}) \mathrm{C}(\mathrm{O}) \mathrm{CO}$ \\
\hline Pinic acid & $\mathrm{CC} 1(\mathrm{C}(\mathrm{CC} 1 \mathrm{C}(=\mathrm{O}) \mathrm{O}) \mathrm{CC}(=\mathrm{O}) \mathrm{O}) \mathrm{C}$ \\
\hline C5-alkene triol & $\mathrm{C}(\mathrm{O}) \mathrm{C}(\mathrm{C})=\mathrm{C}(\mathrm{O}) \mathrm{CO}$ \\
\hline 2-methylglyceric acid & $\mathrm{CC}(\mathrm{CO})(\mathrm{C}(=\mathrm{O}) \mathrm{O}) \mathrm{O}$ \\
\hline Levoglucosan & $\mathrm{C} 1 \mathrm{C} 2 \mathrm{C}(\mathrm{C}(\mathrm{C}(\mathrm{C}(\mathrm{O} 1) \mathrm{O} 2) \mathrm{O}) \mathrm{O}) \mathrm{O}$ \\
\hline Pinonic acid & $\mathrm{CC}(=\mathrm{O}) \mathrm{C} 1 \mathrm{CC}(\mathrm{C} 1(\mathrm{C}) \mathrm{C}) \mathrm{CC}(=\mathrm{O}) \mathrm{O}$ \\
\hline Hydroxyglutaric acid & $\mathrm{C}(\mathrm{CC}(=\mathrm{O}) \mathrm{O}) \mathrm{C}(\mathrm{C}(=\mathrm{O}) \mathrm{O}) \mathrm{O}$ \\
\hline 2-methyltetrol dimer & $\mathrm{OCC}(\mathrm{O})(\mathrm{C}) \mathrm{C}(\mathrm{O}) \mathrm{COC}(\mathrm{CO})(\mathrm{C}) \mathrm{C}(\mathrm{O}) \mathrm{CO}$ \\
\hline
\end{tabular}


Table S4: Saturation concentrations at $\mathrm{T}_{\text {ref }}=298.15 \mathrm{~K}$ and enthalpies of vaporization $(\Delta \mathrm{H}$ in $\mathrm{kJ} / \mathrm{mol})$ for $298.15 \pm 7 \mathrm{~K}$ fitted to reproduce ambient partitioning or predicted based on vapor pressure for the pure species. Fitted values are based on traditional absorptive partitioning to an organic-only medium:

$$
\mathrm{F}_{\mathrm{p}, \mathrm{i}}=\left(1+\mathrm{T}_{\mathrm{ref}} / \mathrm{T} \times \exp \left[\Delta \mathrm{H} / 8314 \mathrm{~kJ}^{-1} \mathrm{~mol} \mathrm{~K} \times\left(1 / \mathrm{T}_{\mathrm{ref}}-1 / \mathrm{T}\right) 1 / \mathrm{K}\right] \times \mathrm{C}^{*} /\left(\mathrm{M}_{\mathrm{i}} \times \mathrm{N}\right)\right)^{-1}
$$

where $\mathrm{M}_{\mathrm{i}}$ is the molecular mass of the species and $\mathrm{N}=\mathrm{C}_{\mathrm{org}} / 200 \mathrm{~g} \mathrm{~mol}^{-1}$. EVAPORATION, MYN, and NN structure-based estimates are provided by UMANSYSPROP (Topping et al., 2016) available at http://umansysprop.seaes.manchester.ac.uk. Lower and upper bound parameter estimates are provided for the $95 \%$ confidence interval of the fits to ambient data. NS indicates the parameter was not statistically significant in the fit. AIOMFAC adjusted $\mathrm{C}^{*}$ reflect base values multiplied by 0.238 (Adj Psat sensitivity calculations).

\begin{tabular}{|c|c|c|c|c|c|c|c|c|}
\hline & 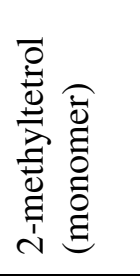 & 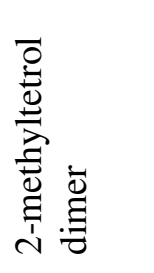 & 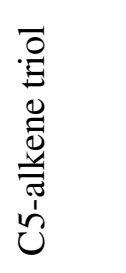 & 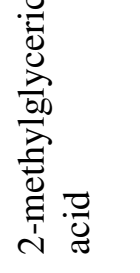 & 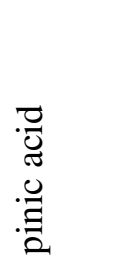 & 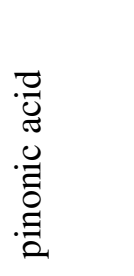 & 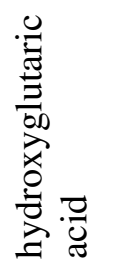 & $\begin{array}{l}\text { चี } \\
0 \\
0 \\
0 \\
0 \\
00 \\
0 \\
0 \\
0\end{array}$ \\
\hline \multicolumn{9}{|l|}{$\mathrm{C}^{*}\left(\mu \mathrm{g} \mathrm{m}^{-3}\right)$} \\
\hline SIMPOL $^{a}$ & 5 & $6.6 \mathrm{E}-07$ & 565 & 4899 & 7 & 980 & 2 & 16 \\
\hline EVAPORATION $^{b}$ & 34 & $2.8 \mathrm{E}-06$ & 63 & 301 & 22 & 7213 & 9 & 18 \\
\hline $\mathrm{MYN}^{\mathrm{c}}$ & 507 & $2.1 \mathrm{E}-01$ & 7217 & 2594 & 1051 & 18366 & 152 & 8172 \\
\hline $\mathrm{NN}^{\mathrm{d}}$ & 10 & $5.8 \mathrm{E}-08$ & 1205 & 115 & 53 & 4556 & 1 & 269 \\
\hline Fit to Ambient & 1.8 & NA & 2.1 & 2.7 & 3.5 & 81 & 0.2 & 0.5 \\
\hline $\begin{array}{l}\text { Fit to Ambient } \\
\text { (lower bound) }\end{array}$ & 1.5 & NA & 1.7 & 2.3 & 3.0 & 70 & 0.2 & 0.4 \\
\hline $\begin{array}{l}\text { Fit to Ambient } \\
\text { (upper bound) }\end{array}$ & 2.1 & NA & 2.5 & 3.2 & 4.2 & 94 & 0.3 & 0.7 \\
\hline $\begin{array}{l}\text { AIOMFAC } \\
\text { Adjusted (Adj Psat) }\end{array}$ & 7.7 & NA & 14 & 69 & 5.1 & 1700 & 2 & 4 \\
\hline \multicolumn{9}{|l|}{$\Delta \mathrm{H}^{\mathrm{vap}}\left(\mathrm{kJ} \mathrm{mol}^{-1}\right)$} \\
\hline SIMPOL & 107 & 167 & 89 & 78 & 99 & 76 & 102 & 98 \\
\hline EVAPORATION & 107 & 176 & 105 & 97 & 112 & 89 & 112 & 115 \\
\hline MYN & 92 & 120 & 83 & 86 & 88 & 78 & 95 & 81 \\
\hline $\mathrm{NN}$ & 117 & 211 & 94 & 106 & 108 & 87 & 127 & 103 \\
\hline Fit to Ambient & 122 & NA & 129 & 71 & 120 & NS & NS & $\mathrm{NS}$ \\
\hline $\begin{array}{l}\text { Fit to Ambient } \\
\text { (lower bound) }\end{array}$ & 87 & NA & 84 & 35 & 84 & NS & NS & NS \\
\hline $\begin{array}{l}\text { Fit to Ambient } \\
\text { (upper bound) }\end{array}$ & 158 & NA & 178 & 108 & 158 & NS & NS & NS \\
\hline
\end{tabular}

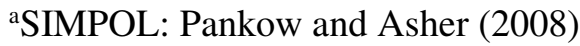

bEVAPORATION: Compernolle et al. (2011). Used with AIOMFAC.

'MYN: Myrdal and Yalkowsky (1997) vapor pressure method with Nanoolal et al. (2004) boiling point method.

${ }^{\mathrm{d}} \mathrm{NN}$ : Nannoolal et al. (2008) vapor pressure method with Nanoolal et al. (2004) boiling point method. 
Table S5: Average concentrations of particulate ammonium and sulfate and their ratios at the SOAS Centreville site from 1 June 2013 to 15 July 2013.

\begin{tabular}{|l|c|c|c|c|c|}
\hline \multicolumn{1}{|c|}{ Instrument } & $\begin{array}{c}\text { Number of } \\
\text { Hourly } \\
\text { Aggregated } \\
\text { Observations }\end{array}$ & $\begin{array}{c}\text { Mean } \\
\text { Ammonium } \\
\left(\mu \mathrm{g} \mathrm{m}^{-3}\right)\end{array}$ & $\begin{array}{c}\text { Mean } \\
\text { Sulfate } \\
\left(\mu \mathrm{g} \mathrm{m}^{-3}\right)\end{array}$ & $\begin{array}{c}\mathrm{R}_{\mathrm{N} / 2 \mathrm{~S}} \\
\text { Molar } \\
\text { Ratio of } \\
\text { Means }\end{array}$ & $\begin{array}{c}\text { Mean of } \\
\text { Molar } \\
\text { Ratio } \\
\mathrm{R}_{\mathrm{N} / 2 \mathrm{~S}}\end{array}$ \\
\hline GT AMS (Xu et al. 2015a,b) $\mathrm{PM}_{1}$ & 881 & 0.40 & 1.8 & 0.59 & 0.51 \\
\hline CU AMS (Hu et al. 2015) $\mathrm{PM}_{1}$ & 646 & 0.39 & 2.2 & 0.47 & 0.44 \\
\hline SEARCH CTR PM 2.5 & 739 & 0.59 & 1.8 & 0.86 & 0.96 \\
\hline MARGA (Allen et al. 2015) $\mathrm{PM}_{2.5}$ & 948 & 0.67 & 2.2 & 0.81 & 0.80 \\
\hline $\begin{array}{l}\text { URG Corporation Ambient Ion } \\
\text { Monitor (AIM) 9000-D PM } \text { P\&2.5 }_{1 \& 2}\end{array}$ & 374 & 0.91 & 2.1 & 1.2 & 1.4 \\
\hline
\end{tabular}

Table S6: Molar ratio of ammonium to sulfate $\left(\mathrm{R}_{\mathrm{N} / \mathrm{S}}\right)$ from Silvern et al. (2017) and resulting $\mathrm{R}_{\mathrm{N} / 2 \mathrm{~S}}$.

\begin{tabular}{|l|c|c|}
\hline Dataset & $\mathrm{R}_{\mathrm{N} / \mathrm{S}}$ & $\mathrm{R}_{\mathrm{N} / 2 \mathrm{~S}}$ \\
\hline Eastern US CSN Summer 2013 $\mathrm{PM}_{2.5}$ & 1.44 & 0.72 \\
\hline CU AMS at SOAS CTR PM & 0.93 & 0.47 \\
\hline AMS on SEAC ${ }^{4}$ RS aircraft (RMA regression) $\mathrm{PM}_{1}$ & 1.21 & 0.60 \\
\hline SEARCH (five site mean) $\mathrm{PM}_{2.5}$ & 1.62 & 0.81 \\
\hline
\end{tabular}

Table S7: Average concentration of ammonia at the SOAS Centreville site from 1 June 2013 to 15 July 2013. ppb to $\mu \mathrm{g} \mathrm{m}^{-3}$ conversions assume $303.15 \mathrm{~K}\left(1 \mathrm{ppb}=0.68 \mu \mathrm{g} \mathrm{m}^{-3}\right)$.

\begin{tabular}{|l|c|c|c|c|}
\hline \multicolumn{1}{|c|}{ Instrument } & $\begin{array}{c}\text { Number of Hourly } \\
\text { Aggregated Observations }\end{array}$ & $\begin{array}{c}\text { Ammonia } \\
(\mathrm{ppb})\end{array}$ & $\begin{array}{c}\text { Ammonia } \\
\left(\mu \mathrm{g} \mathrm{m}^{-3}\right)\end{array}$ & $\begin{array}{c}\text { Ratio of } \\
\text { Means: } \\
\mathrm{NH}_{4}{ }^{+} / \mathrm{NH}_{\mathrm{x}}\end{array}$ \\
\hline SEARCH CTR & 915 & 0.38 & 0.26 & 0.68 \\
\hline MARGA (Allen et al., 2015) & 948 & 0.75 & 0.51 & 0.55 \\
\hline CIMS (You et al., 2014) & 799 & 0.52 & 0.36 & $\mathrm{NA}$ \\
\hline $\begin{array}{l}\text { URG Corporation Ambient } \\
\text { Ion Monitor (AIM) 9000-D }\end{array}$ & 370 & 0.85 & 0.58 & 0.50 \\
\hline
\end{tabular}


Table S8: Mean $\mathrm{C}^{*}$ accounting for the effects of temperature and ideality in CLLPS and EQLB and for pure the species at $298.15 \mathrm{~K}$ (Adj Psat, adjusted vapor pressure calculations). For AIOMFAC

calculations, $C^{*}$ follows equation 4 . Thus, for a system with two liquid phases ( $\alpha$ and $\beta$ ) in the particle $(\mathrm{PM})$, the following results:

$$
C_{i}^{*}=\frac{P_{i}^{s a t} \gamma_{i}^{\alpha}\left(\sum_{k} C_{k}^{P M}\right)}{R T\left(\sum_{k} C_{k}^{\alpha} / M_{k}\right)}\left(\frac{C_{i}^{\alpha}}{C_{i}^{\alpha}+C_{i}^{\beta}}\right)
$$

where $P_{i}^{\text {sat }}$ is the pure species vapor pressure at temperature T, $\gamma_{i}^{\alpha}$ is the mole-fraction based activity coefficient for species $i$ in the $\alpha$ phase, $C_{i}^{\alpha}$ is the mass concentration of species $i$ in the $\alpha$ phase, $C_{i}^{\beta}$ is the mass concentration of species $i$ in the $\beta$ phase, $M_{k}$ is the molecular mass of species $k$, and the summations are over all PM species (water, organic compounds, and inorganic compounds). The $C_{i}^{*}$ could be defined analogously for the $\beta$ phase. For one liquid phase, the equation reduces to:

$$
C_{i}^{*}=\frac{P_{i}^{s a t} \gamma_{i} M_{P M}}{R T}
$$

where the effective PM molecular mass $\left(M_{P M}\right)$ is:

$$
M_{P M}=\frac{\sum_{k} C_{k}^{P M}}{\sum_{k} C_{k}^{P M} / M_{k}}
$$

\begin{tabular}{|l|r|r|r|r|r|}
\hline \multicolumn{1}{|c|}{ species } & $\begin{array}{c}\text { CLLPS C* } \\
\left(\mu \mathrm{g} \mathrm{m}^{-3}\right)\end{array}$ & $\begin{array}{c}\text { EQLB C* } \\
\left(\mu \mathrm{g} \mathrm{m}^{-3}\right)\end{array}$ & $\begin{array}{c}\text { Pure Species } \\
\mathrm{C}^{*}\left(\mu \mathrm{g} \mathrm{m}^{-3}\right)\end{array}$ & $\begin{array}{c}\text { Ratio } \\
\text { EQLB C*/ } \\
\text { CLLPS C* }\end{array}$ & $\begin{array}{c}\text { Ratio } \\
\text { EQLB C*/ } \\
\text { Pure C* }\end{array}$ \\
\hline 2-methyltetrol & 6.0 & 3.7 & 7.7 & 0.62 & 0.47 \\
\hline pinic acid & 13 & 16 & 5.1 & 1.19 & 3.09 \\
\hline C $_{5}$-alkene triol & 22 & 17 & 14 & 0.78 & 1.19 \\
\hline 2-methylglyceric acid & 43 & 22 & 69 & 0.50 & 0.31 \\
\hline levoglucosan & 1.6 & 1.4 & 4 & 0.90 & 0.35 \\
\hline pinonic acid & $2.0 \mathrm{E}+04$ & $3.1 \mathrm{E}+04$ & $1.7 \mathrm{E}+03$ & 1.55 & 18.7 \\
\hline hydroxyglutaric acid & 0.85 & 0.60 & 2 & 0.71 & 0.29 \\
\hline
\end{tabular}

Table S9: Mean activity coefficients predicted by AIOMFAC (mole-fraction based) for semivolatile organics (Adj Psat calculations). The $\beta$ phase was organic-rich in both CLLPS and EQLB calculations.

\begin{tabular}{|l|r|r|r|r|}
\hline \multicolumn{1}{|c|}{ species } & \multicolumn{1}{c|}{$\begin{array}{c}\gamma \text { CLLPS } \\
\beta \text { phase }\end{array}$} & $\begin{array}{c}\gamma \text { EQLB } \\
\beta \text { phase }\end{array}$ & $\begin{array}{c}\gamma \text { EQLB } \\
\alpha \text { phase }\end{array}$ & $\begin{array}{c}\text { Ratio: } \\
\gamma_{\beta} \text { EQLB } \gamma_{\beta} \text { CLLPS }\end{array}$ \\
\hline 2-methyltetrol & 0.63 & 0.77 & $4.7 \mathrm{E}+03$ & 1.23 \\
\hline pinic acid & 5.22 & 16.21 & $1.4 \mathrm{E}+09$ & 3.10 \\
\hline C5-alkene triol $_{5}$-methylglyceric acid & 1.37 & 2.04 & $9.3 \mathrm{E}+04$ & 0.97 \\
\hline 2-m & 0.49 & 0.48 & 23 & 2.45 \\
\hline levoglucosan & 0.42 & 1.02 & $1.4 \mathrm{E}+05$ & 4.56 \\
\hline pinonic acid & 26.60 & 121.31 & $1.3 \mathrm{E}+10$ & 2.63 \\
\hline hydroxyglutaric acid & 0.36 & 0.96 & 290 & 1.49 \\
\hline
\end{tabular}




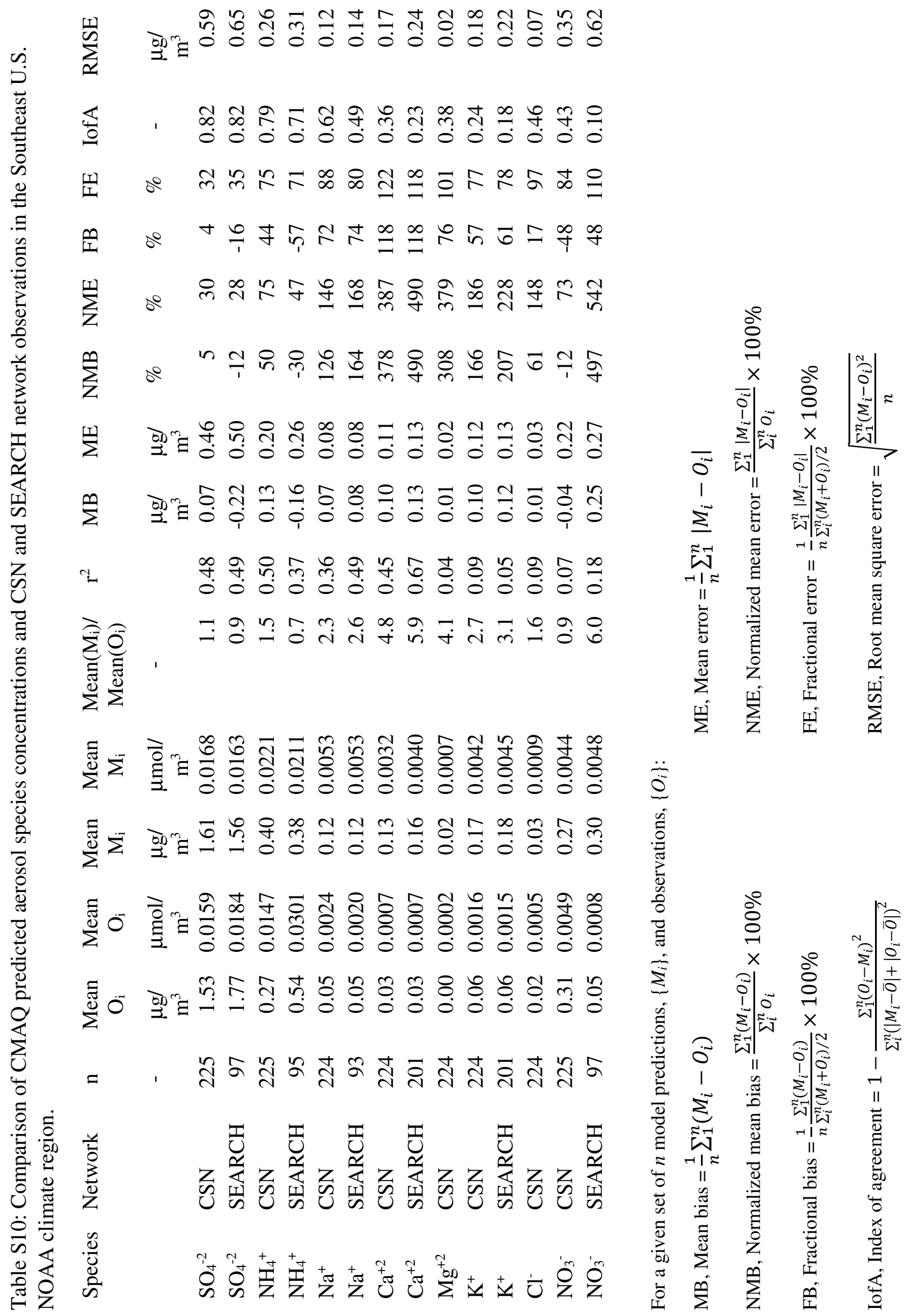


Figure S1: Observed (CSN, IMPROVE) and modeled (CMAQ) ions for June 1, 2013 to July 15, 2013.

Major cations and anions for the Southeast U.S. NOAA Climate Region (FL, GA, SC, NA, VA)

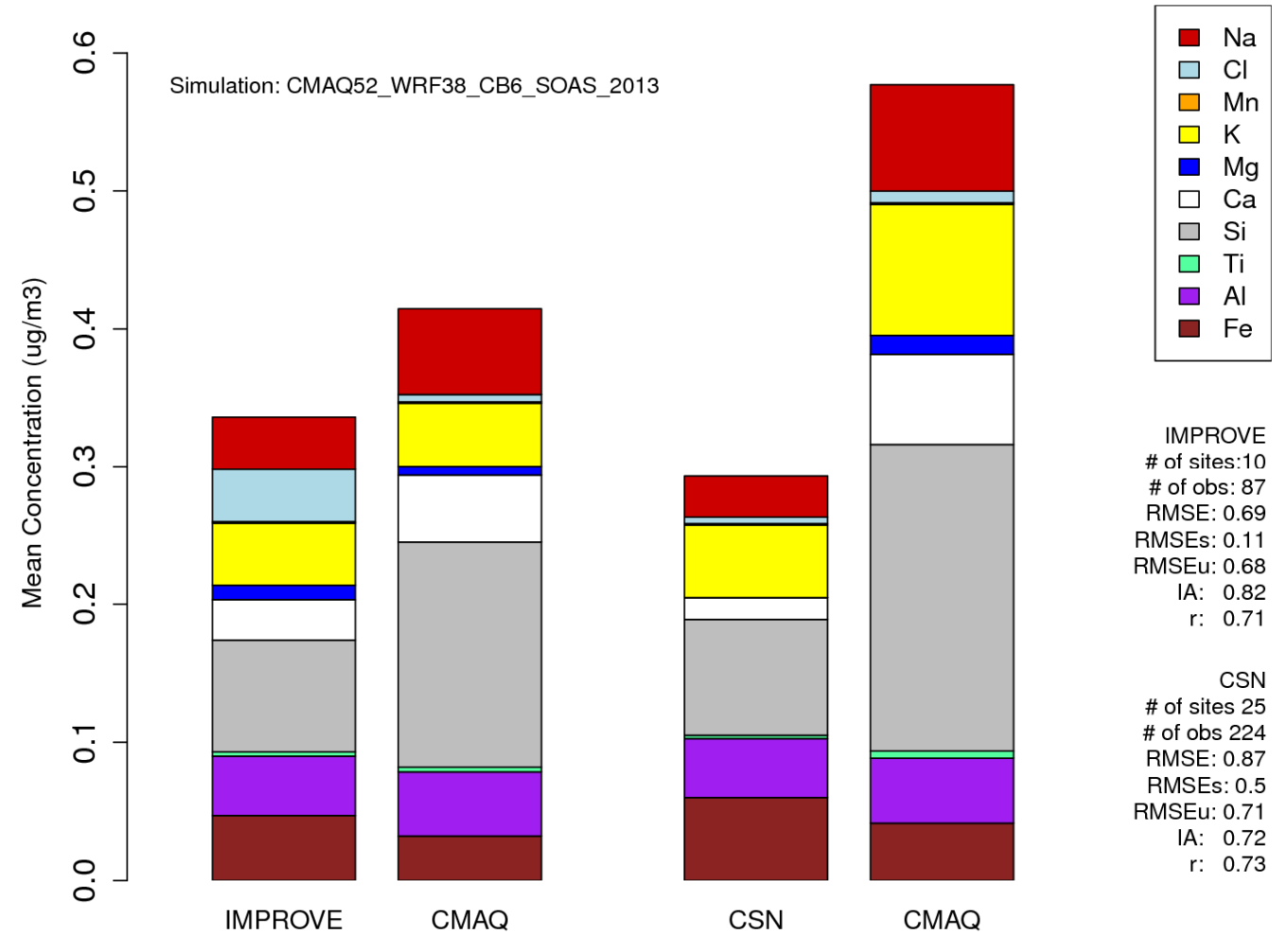


Figure S2: Observed (CSN-circle, SEARCH-triangle) and modeled (CMAQ) ammonium for June 1, 2013 to July 15, 2013. Ammonium is not measured by the IMPROVE network.

(a) Observed Ammonium $\left(\mu \mathrm{g} \mathrm{m}^{-3}\right)$

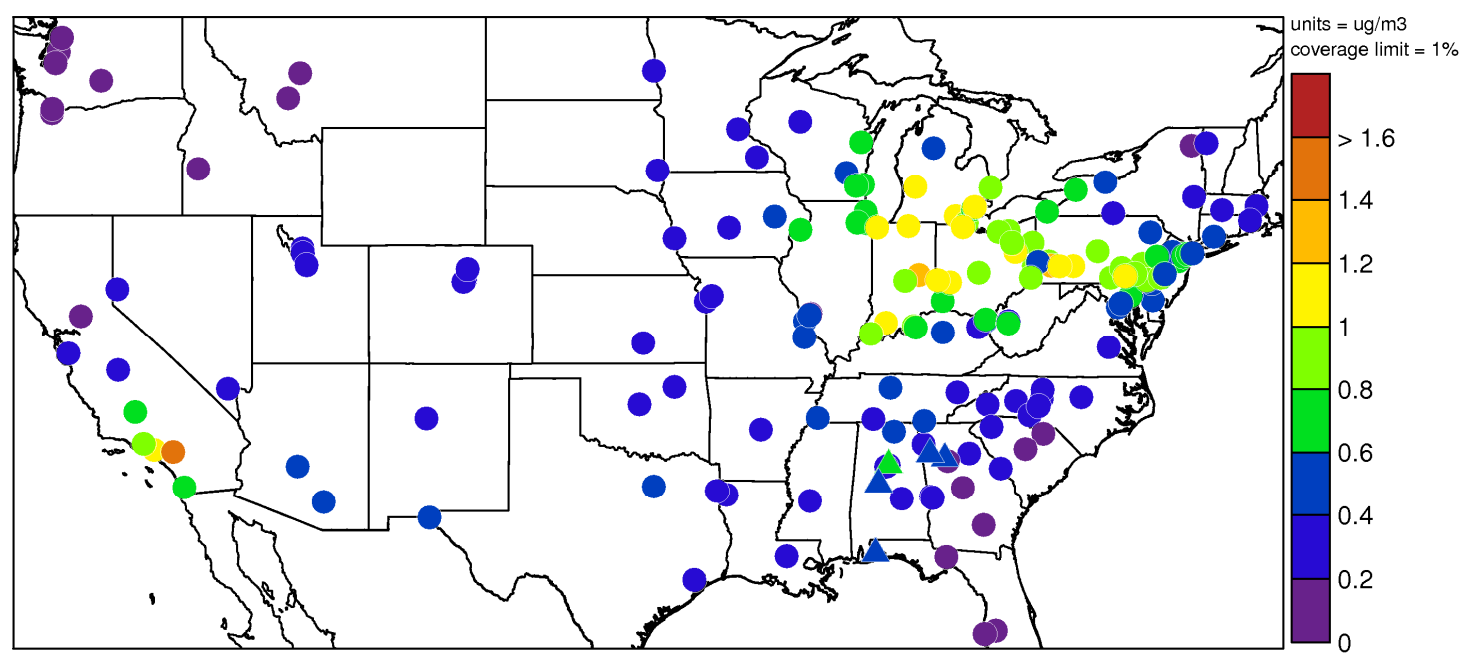

(b) Modeled - Observed Ammonium $\left(\mu \mathrm{g} \mathrm{m} \mathrm{m}^{-3}\right)$

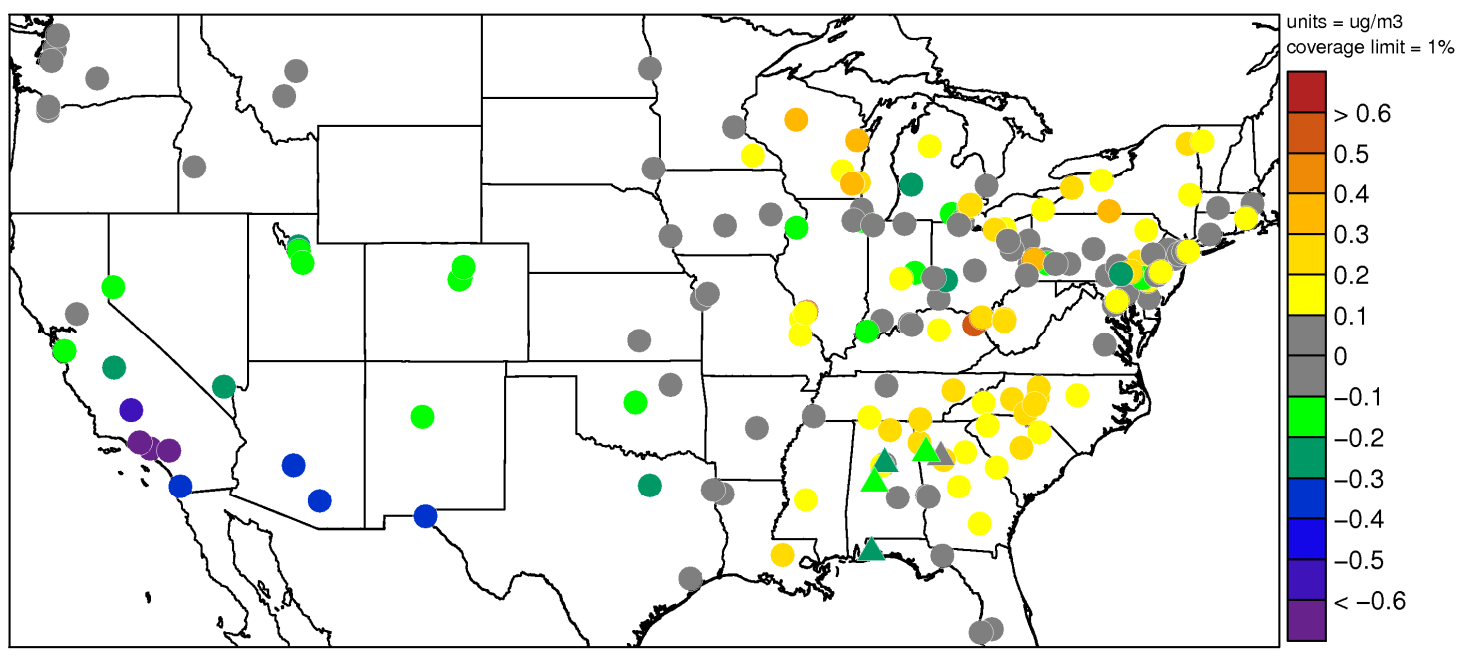


Figure S3: Observed (IMPROVE-square, CSN-circle, SEARCH-triangle) and modeled (CMAQ) sulfate for June 1, 2013 to July 15, 2013.

(a) Observed sulfate $\left(\mu \mathrm{g} \mathrm{m}^{-3}\right)$

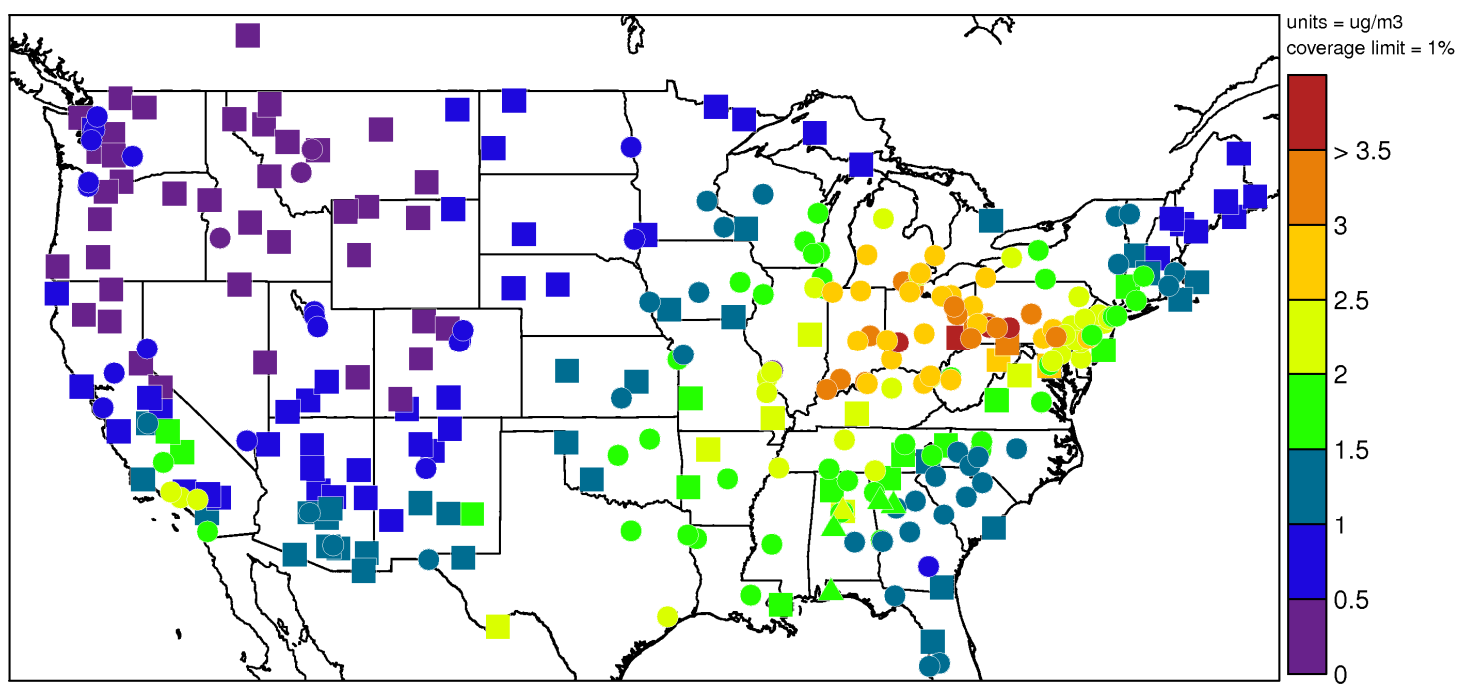

(b) Modeled - Observed sulfate $\left(\mu \mathrm{g} \mathrm{m}^{-3}\right)$

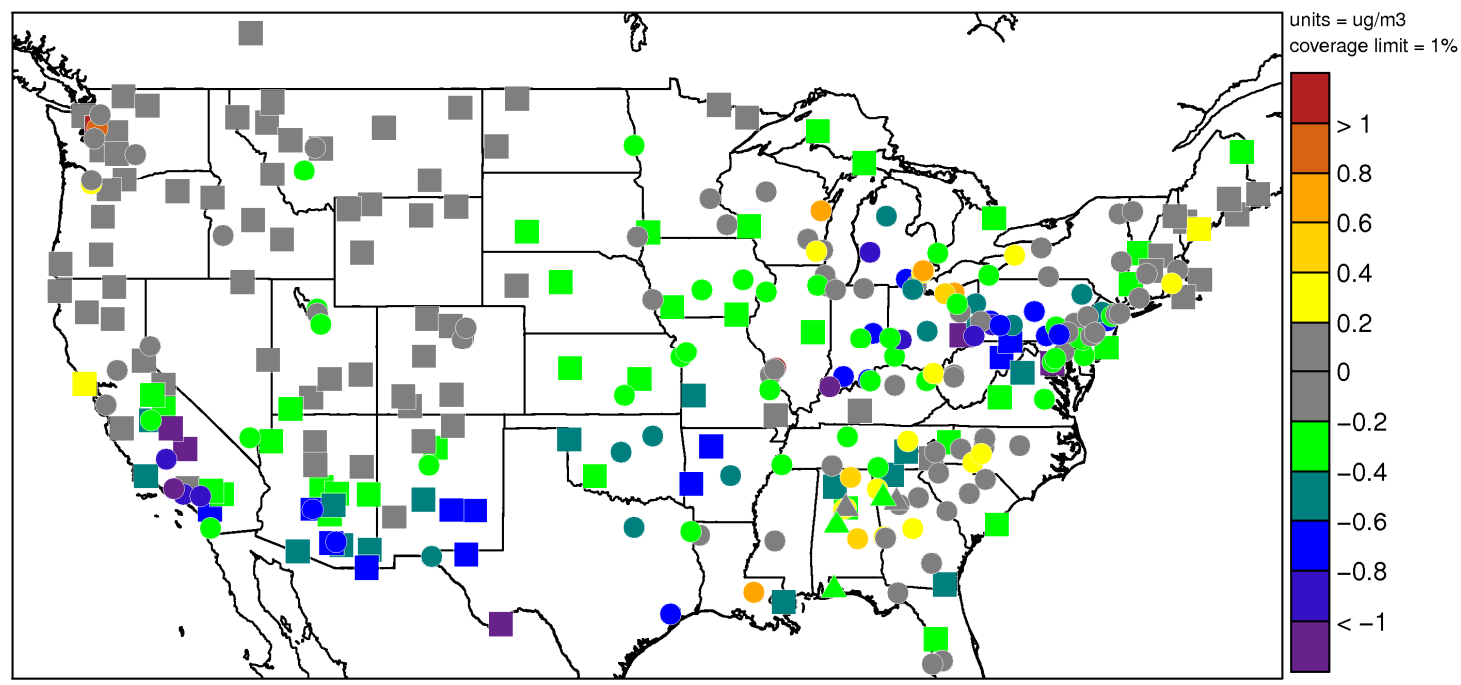


Figure S4: Modeled vs Observed (CSN) Molar Ratio of (a) ammonium to $2 \times$ sulfate and (b) cations to anions $(2 \times$ calcium + potassium + sodium + ammonium $+2 \times$ magnesium $) /(2 \times$ sulfate + nitrate + chloride).

(a) Ratio of ammonium to $2 \times$ sulfate

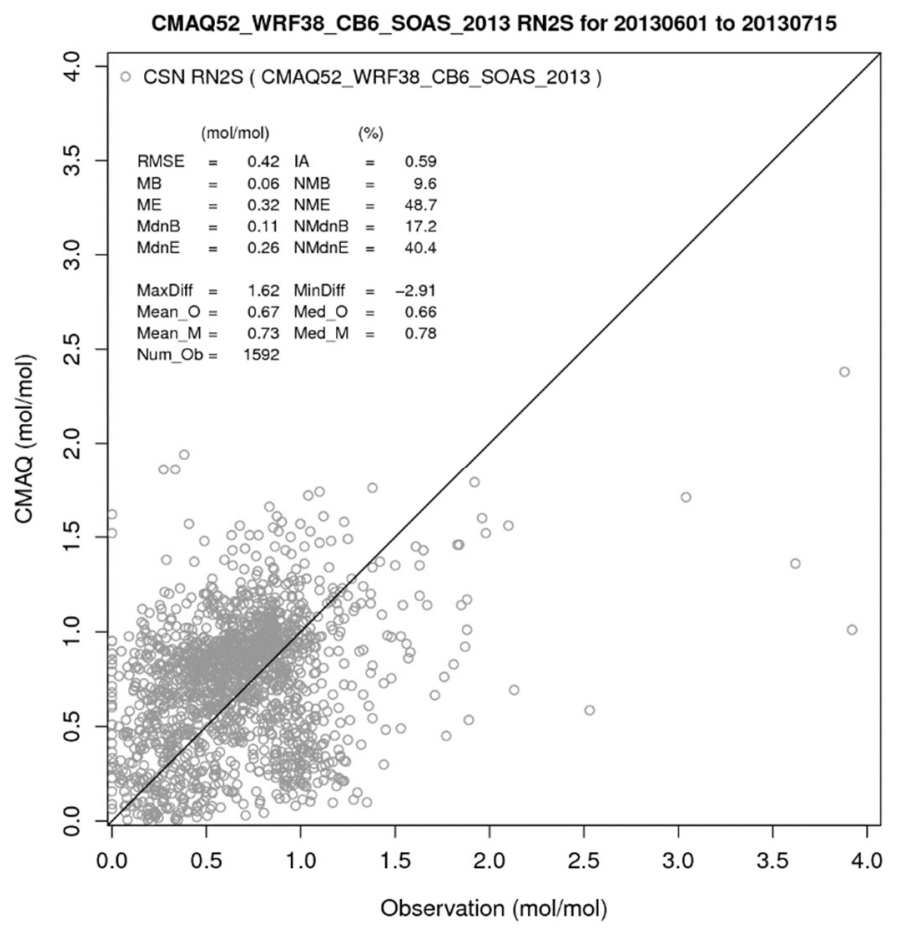

(b) Ratio of cations to anions

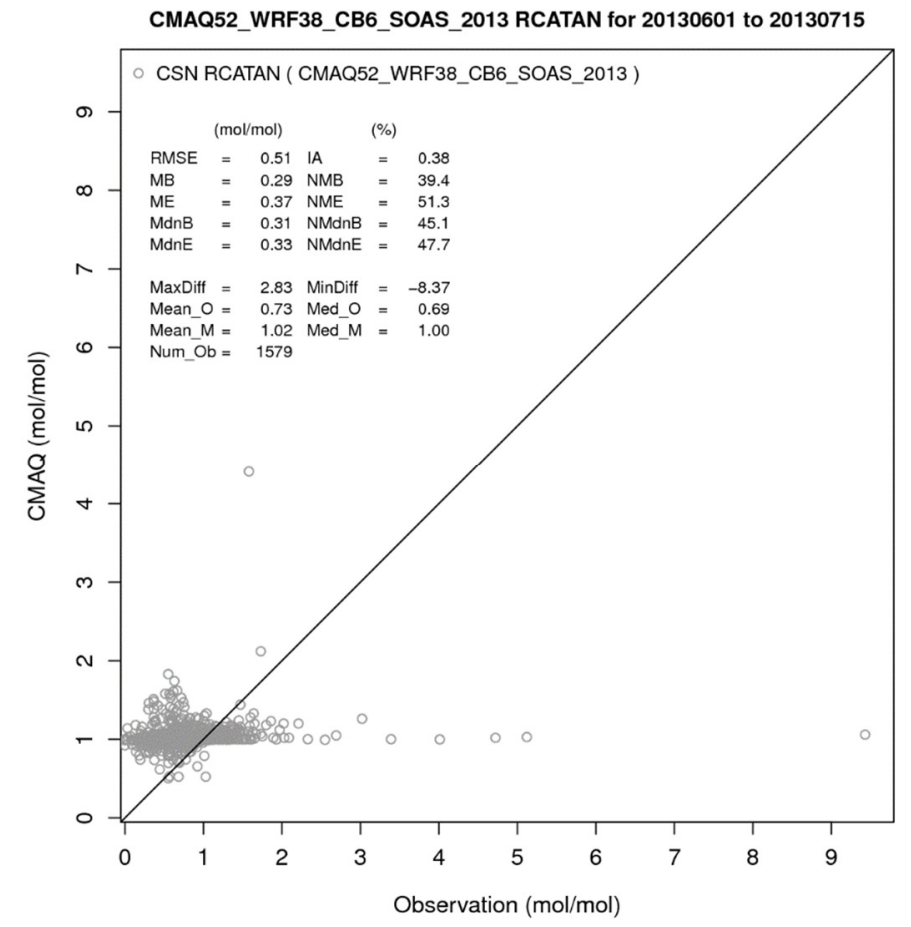


Figure S5: (a) Observed (Ammonia monitoring Network, AMoN), (b) CMAQ simulated, and (c) model bias in gas-phase ammonia concentrations June 1, 2013- July 15, 2013.

(a) AMoN Ammonia (ppb)

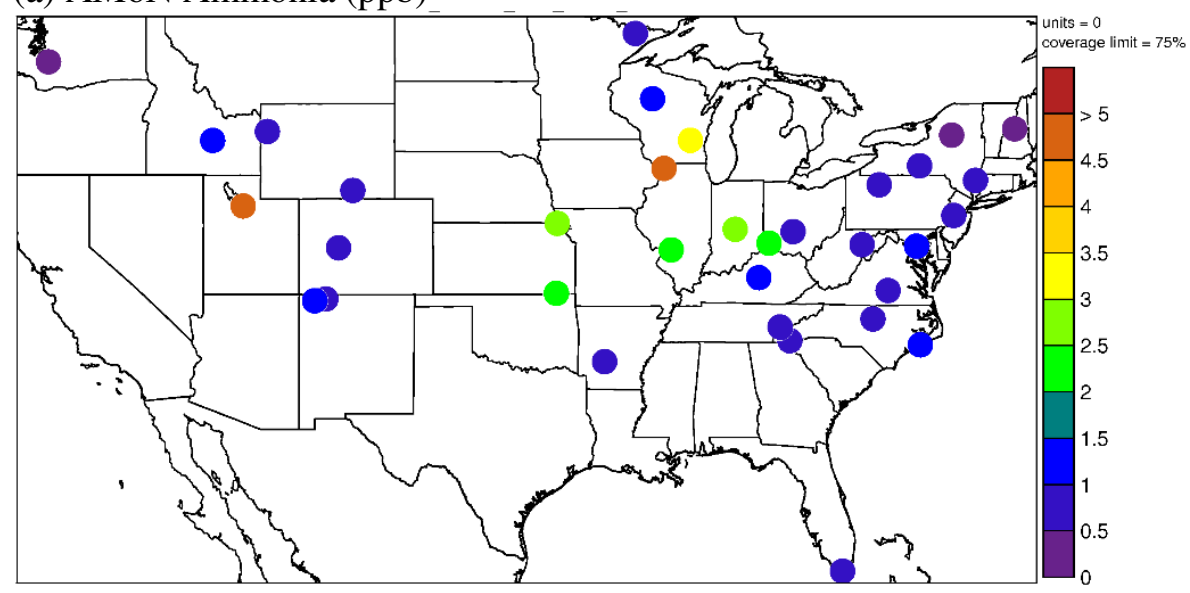

(b) CMAQ Predicted Ammonia (ppb)

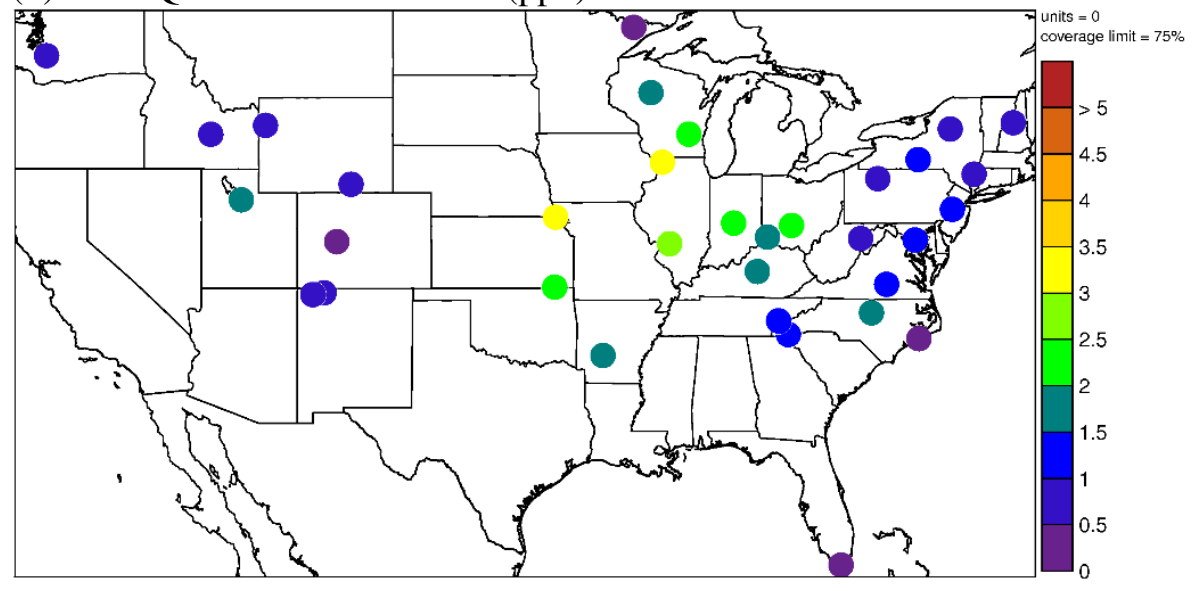

(c) Modeled - Observed Ammonia (ppb)

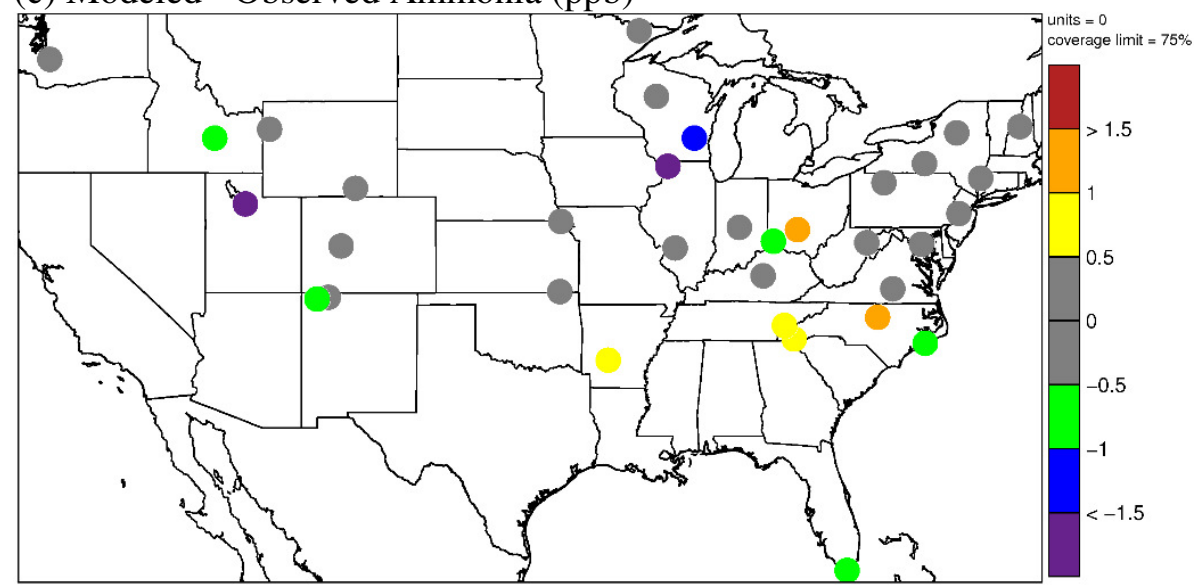


Figure S6: Observed and CMAQ predicted inorganic species at SOAS Centreville site.

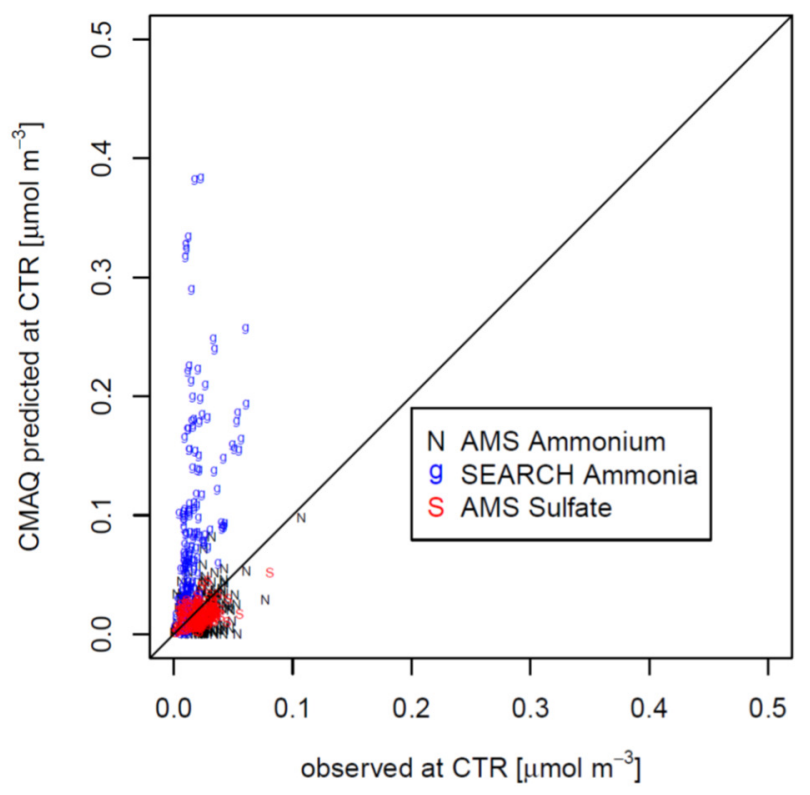

Figure S7: Liquid-liquid phase separation as a function of hour of day predicted by AIOMFAC for the ammonium-sodium-sulfate-nitrate-chloride and organic surrogates system. Shown is the percentage of the time a phase separation was predicted in a certain hour-of-day bin. For reference, the oxygen-to-carbon ratio based separation relative humidity (SRH) as parameterized by You et al. (2013) is shown in blue.

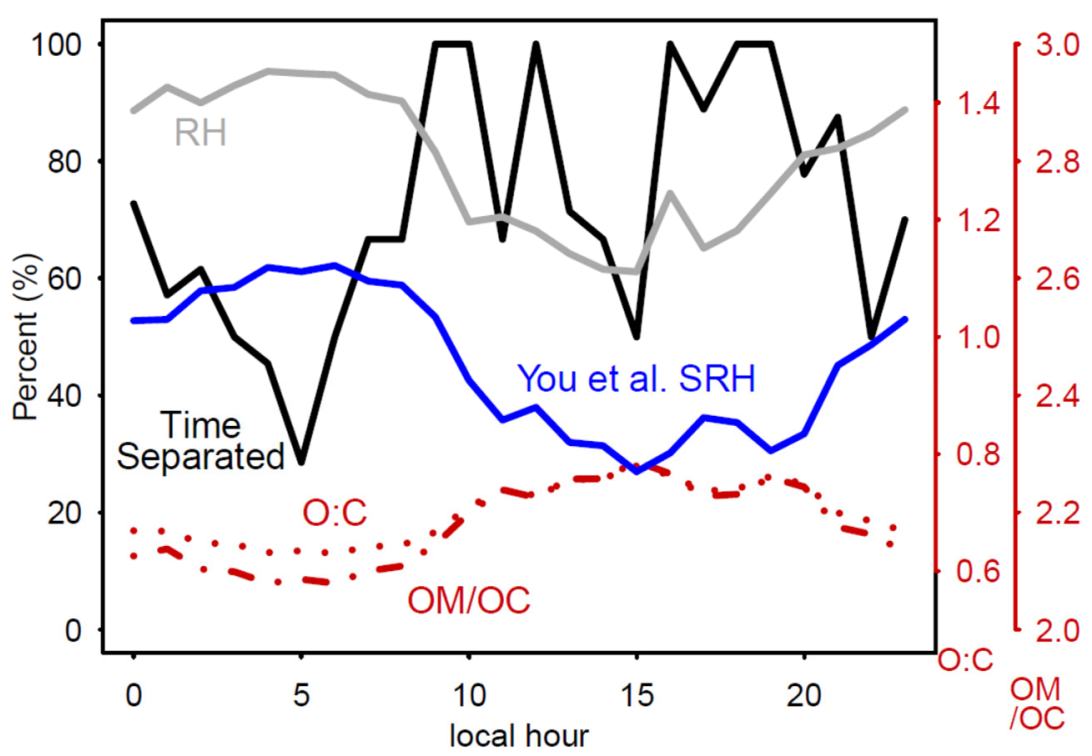


Figure S8: $\mathrm{r}^{2}$ (square of Pearson's $\mathrm{r}$ ) between model predicted and observed $\mathrm{F}_{\mathrm{p}}$ for each explicit semivolatile species. The $\mathrm{x}$-axis location is arbitrary for the Traditional regression (equation $\mathrm{S} 1$ ). $\mathrm{r}^{2}$ does not exceed 0.25 for any species or method.

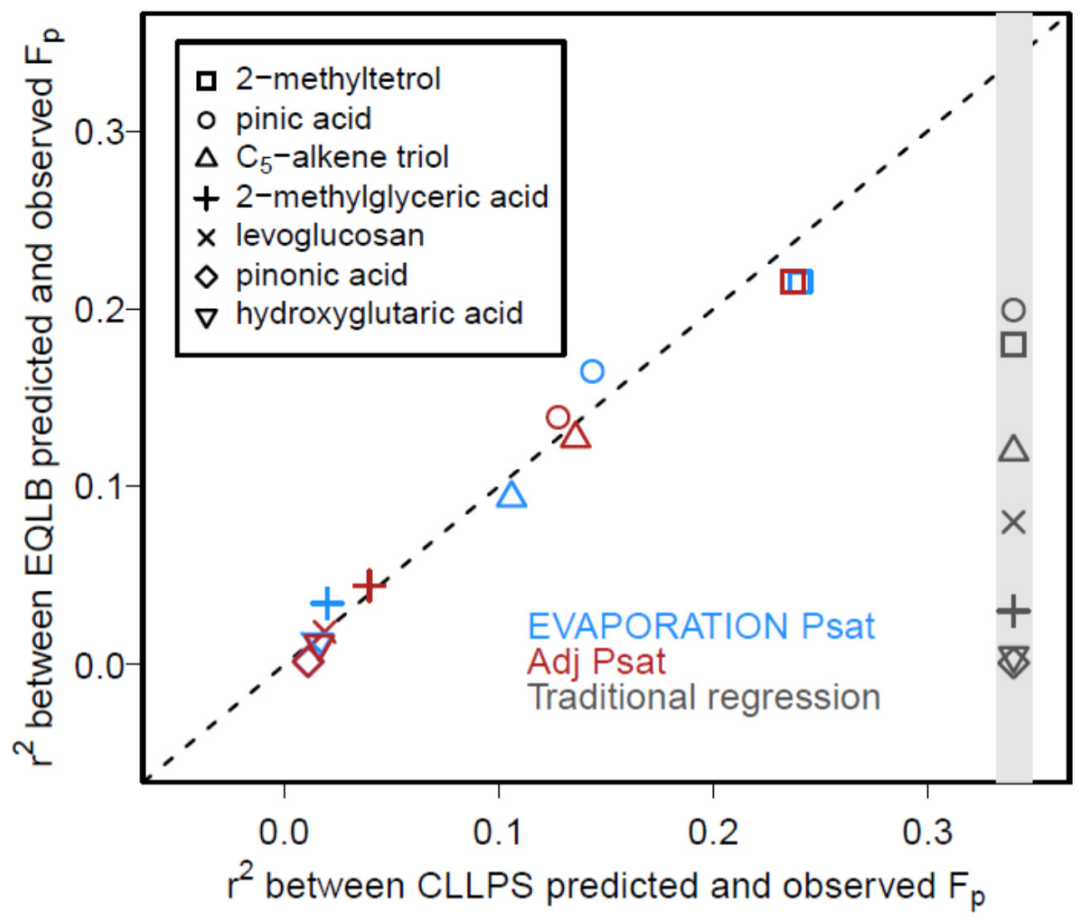




\section{References}

Allen, H. M., Draper, D. C., Ayres, B. R., Ault, A., Bondy, A., Takahama, S., Modini, R. L., Baumann, K., Edgerton, E., Knote, C., Laskin, A., Wang, B., and Fry, J. L.: Influence of crustal dust and sea spray supermicron particle concentrations and acidity on inorganic $\mathrm{NO}_{3}{ }^{-}$aerosol during the 2013 Southern Oxidant and Aerosol Study, Atmos. Chem. Phys., 15, 10669-10685, doi: 10.5194/acp-15-10669-2015, 2015.

Compernolle, S., Ceulemans, K., and Müller, J. F.: EVAPORATION: a new vapour pressure estimation methodfor organic molecules including non-additivity and intramolecular interactions, Atmos. Chem. Phys., 11, 9431-9450, doi: 10.5194/acp-11-9431-2011, 2011.

Hu, W. W., Campuzano-Jost, P., Palm, B. B., Day, D. A., Ortega, A. M., Hayes, P. L., Krechmer, J. E., Chen, Q., Kuwata, M., Liu, Y. J., de Sá, S. S., McKinney, K., Martin, S. T., Hu, M., Budisulistiorini, S. H., Riva, M., Surratt, J. D., St. Clair, J. M., Isaacman-Van Wertz, G., Yee, L. D., Goldstein, A. H., Carbone, S., Brito, J., Artaxo, P., de Gouw, J. A., Koss, A., Wisthaler, A., Mikoviny, T., Karl, T., Kaser, L., Jud, W., Hansel, A., Docherty, K. S., Alexander, M. L., Robinson, N. H., Coe, H., Allan, J. D., Canagaratna, M. R., Paulot, F., and Jimenez, J. L.: Characterization of a real-time tracer for isoprene epoxydiols-derived secondary organic aerosol (IEPOX-SOA) from aerosol mass spectrometer measurements, Atmos. Chem. Phys., 15, 11807-11833, doi: 10.5194/acp-15-11807-2015, 2015.

Myrdal, P. B., and Yalkowsky, S. H.: Estimating pure component vapor pressures of complex organic molecules, Ind. Eng. Chem. Res., 36, 2494-2499, doi: 10.1021/ie9502421, 1997.

Nannoolal, Y., Rarey, J., Ramjugernath, D., and Cordes, W.: Estimation of pure component properties: Part 1. Estimation of the normal boiling point of non-electrolyte organic compounds via group contributions and group interactions, Fluid Phase Equilibr., 226, 45-63, doi: 10.1016/j.fluid.2004.09.001, 2004.

Nannoolal, Y., Rarey, J., and Ramjugernath, D.: Estimation of pure component properties: Part 3. Estimation of the vapor pressure of non-electrolyte organic compounds via group contributions and group interactions, Fluid Phase Equilibr., 269, 117-133, doi: 10.1016/j.fluid.2008.04.020, 2008.

Pankow, J. F., and Asher, W. E.: SIMPOL.1: a simple group contribution method for predicting vapor pressures and enthalpies of vaporization of multifunctional organic compounds, Atmos. Chem. Phys., 8, 2773-2796, doi: 10.5194/acp-8-2773-2008, 2008.

Silvern, R. F., Jacob, D. J., Kim, P. S., Marais, E. A., Turner, J. R., Campuzano-Jost, P., and Jimenez, J. L.: Inconsistency of ammonium-sulfate aerosol ratios with thermodynamic models in the eastern US: a possible role of organic aerosol, Atmos. Chem. Phys., 17, 5107-5118, doi: 10.5194/acp-17-5107-2017, 2017.

Topping, D., Barley, M., Bane, M. K., Higham, N., Aumont, B., Dingle, N., and McFiggans, G.: UManSysProp v1.0: an online and open-source facility for molecular property prediction and atmospheric aerosol calculations, Geosci. Model Dev., 9, 899-914, doi: 10.5194/gmd-9-899-2016, 2016.

Xu, L., Guo, H., Boyd, C. M., Klein, M., Bougiatioti, A., Cerully, K. M., Hite, J. R., Isaacman-VanWertz, G., Kreisberg, N. M., Knote, C., Olson, K., Koss, A., Goldstein, A. H., Hering, S. V., de Gouw, J., Baumann, K., Lee, S.-H., Nenes, A., Weber, R. J., and Ng, N. L.: Effects of anthropogenic emissions on aerosol formation from isoprene and monoterpenes in the southeastern United States, P. Natl. Acad. Sci. USA, 112, 37-42, doi: 10.1073/pnas.1417609112, 2015a. 
Xu, L., Suresh, S., Guo, H., Weber, R. J., and Ng, N. L.: Aerosol characterization over the southeastern United States using high-resolution aerosol mass spectrometry: spatial and seasonal variation of aerosol composition and sources with a focus on organic nitrates, Atmos. Chem. Phys., 15, 7307-7336, doi:10.5194/acp-15-7307-2015, 2015 b.

You, Y., Renbaum-Wolff, L., and Bertram, A. K.: Liquid-liquid phase separation in particles containing organics mixed with ammonium sulfate, ammonium bisulfate, ammonium nitrate or sodium chloride, Atmos. Chem. Phys., 13, 11723-11734, doi: 10.5194/acp-13-11723-2013, 2013.

You, Y., Kanawade, V. P., de Gouw, J. A., Guenther, A. B., Madronich, S., Sierra-Hernández, M. R., Lawler, M., Smith, J. N., Takahama, S., Ruggeri, G., Koss, A., Olson, K., Baumann, K., Weber, R. J., Nenes, A., Guo, H., Edgerton, E. S., Porcelli, L., Brune, W. H., Goldstein, A. H., and Lee, S. H.: Atmospheric amines and ammonia measured with a chemical ionization mass spectrometer (CIMS), Atmos. Chem. Phys., 14, 12181-12194, doi: 10.5194/acp-14-12181-2014, 2014.

Zuend, A., and Seinfeld, J. H.: Modeling the gas-particle partitioning of secondary organic aerosol: the importance of liquid-liquid phase separation, Atmos. Chem. Phys., 12, 3857-3882, doi: 10.5194/acp-123857-2012, 2012. 